\title{
Electronic Structure of Strained GaSb/GaAs Quantum Dot: Tight-Binding Approach
}

\author{
W. Sukkabot, Member, IACSIT
}

\begin{abstract}
GaSb quantum dots (QDs) in a GaAs matrix have attracted much attention for the potential ability to extend the accessible wavelength of QD emitters towards a wavelength of > $1.3 \mu \mathrm{m}$ owing to their unique electronic and optical properties caused by their staggered (type-II) band alignment. North $e t$ al. calculated the electronic structure of self-assembled $\mathrm{GaSb} / \mathrm{GaAs}$ dots by using the simple one-band model (effective mass model). Therefore it is interesting to carry out the detailed calculations using a more accurate method, in particular in the view of the new experiments. The aim of this work is to study the electronic properties of $\mathrm{GaSb} / \mathrm{GaAs}$ self-assembled quantum dots (SAD) by using the $\mathrm{sp}^{3} \mathrm{~s}^{*}$ empirical tight-binding method. The influence of strain on the electronic structure is determined by minimizing the elastic energy within the Valence Force Field Approach and the piezoelectric potential is calculated by solving the corresponding 3D Poisson equation. Single-particle bound-state energies are computed as a function of the dot sizes. Finally we have found that the theoretical PL spectra are in a good agreement with the experiment and k.p method.
\end{abstract}

Index Terms-Tight-binding method, quantum dot, strain distribution and piezoelectricity.

\section{INTRODUCTION}

Quantum dots have attracted much attention due to the technological applications in optoelectronic devices, such as Quantum Dot lasers. The strong localization of the electronic wave function in the quantum dots leads to an atomic-like electronic structure and to the possibility of novel and improved photonic and electronic devices. In particular, type-II quantum dots are attracted the strong interest because of their special band structure when either electrons or holes are confined in the dots. The potential application of type-II quantum dots is to extend the accessible wavelength $>1.3 \mu \mathrm{m}$ for light emitting devices based on $\mathrm{GaSb} / \mathrm{GaAs}$ quantum dot [1]-[5].

Due to the lattice mismatch between quantum dot and surrounding material, the strain effects are substantial in these quantum dots. We employ the atomistic calculations, Valence Force Field approach [6]-[8], to find the relaxed atomic positions in this structure. Various theoretical approaches are used to study the quantum dots, ranging from first-principle calculations to empirical models. Due to the high computational demand of the first-principle calculations,

Manuscript received January 12, 2013; revised March 24, 2013.

W. Sukkabot is with Department of Physics Faculty of Science, Ubon Ratchathani University, Ubon Ratchathani, 34190 Thailand (e-mail: w.sukkabot@gmail.com). empirical models are widely implemented to study a large quantum dots. There are three empirical models, the k.p approximation [9], the pseudopotential model [10] and the tight-binding model [11], [12]. The k.p approximation treats a quantum dot as a confined bulk and continuum system, while the pseudopotential and tight-binding models treat the system with the atomistic description. The distinction between the two atomistic models is the degree of atomic detail included in the model. Within the tight-binding model, the atomistic detail is limited to a small basis set, while in the pseudopotential model the feature of wave functions is described with a large basis set. Therefore the tight-binding model is computationally less costly than the pseudopotential model. The tight-binding model is a good candidate for the study of relatively big and complicated systems.

At the moment, the theoretical calculations of the type-II quantum dots have not been extensively investigated and there are only few papers that we can mention here. North and co-authors [13] investigated the electronic structure of type-II GaSb/GaAs quantum dots by using the multiband effective-mass calculation. K. Gradkowski and et al. [2] reported the results of the room temperature photoreflectance (PR) and photoluminescence (PL) measurements of molecular beam epitaxy (MBE) grown GaAsSb/GaAs quantum dot structure: one with a capping quantum well and one without it. PL technique is employed to probe the ground-state transition energies. Using the experimental data, they calculated a band structure of these dots by using the 8-band k.p approach. The theoretical analysis accounts well in both $\mathrm{GaAsSb} / \mathrm{GaAs}$ quantum dot with a capping quantum well and one without it.

In this paper, we report the tight-binding calculations of the type-II GaSb/GaAs quantum dots. The strain distribution of the quantum dot is calculated by using the Valence Force Field method. The piezoelectricity is also obtained by solving the 3D Poisson's equation. Then we numerically calculate the electronic structure of the quantum dot as a function of the dot sizes. Finally we compare our calculated results with the experiment and another numerical analysis.

\section{THEORY}

\section{A. Valence Force Field Method (VFF)}

In a realistic structure, the quantum dot and surrounding material are fabricated from two types of the materials. Each material has its own lattice constant. Therefore it leads to the lattice-mismatch-induced strain in this structure. To calculate the atomic structure of the $\mathrm{GaSb} / \mathrm{GaAs}$ quantum dot, the strain relaxation has been calculated. In this work, we 
implement the Valence Force Field method [6]-[8] to find the relaxed structure. The elastic energy of all atoms is expressed as a function of the atomic positions $R_{i}$ as

$$
\begin{aligned}
& E=\sum_{i} \sum_{j=1}^{4} \frac{3 \alpha_{i j}}{16\left(d_{i j}^{0}\right)^{2}}\left[\left(R_{j}-R_{i}\right)^{2}-\left(d_{i j}^{0}\right)^{2}\right]^{2}+ \\
& \sum_{i} \sum_{j, k>1}^{4} \frac{3 \beta_{i j k}}{8 d_{i j}^{0} d_{i k}^{0}}\left[\left(R_{j}-R_{i}\right) \cdot\left(R_{k}-R_{i}\right)-\cos \theta d_{i j}^{0} d_{i k}^{0}\right]^{2}
\end{aligned}
$$

Here, $d_{i j}^{0}$ denotes the bulk equilibrium bond length between nearest-neighbour atom $i$ and $j$ in the corresponding binary compound, and $\theta=\arccos (1 / 3)$ is the ideal bond angle.

The first term is a sum over all atom $i$ and it nearest neighbours $j$. The second term is a sum over all atoms $i$ and its distinct pairs of its nearest neighbours $j$ and $k$. The fitting dependent parameters $\alpha$ and $\beta$ are the bond-stretching and bond-bending force constants, respectively. For GaAs material, we use $d_{i j}^{0}=2.448 \AA, \quad \alpha=41.49 \times 10^{3} \mathrm{dyne} / \mathrm{cm}$, $\beta=8.94 \times 10^{3} \mathrm{dyne} / \mathrm{cm}$ [14] for GaSb material, we use $d_{i j}^{0}=2.640 \stackrel{\circ}{\AA}, \alpha=33.16 \times 10^{3} \mathrm{dyne} / \mathrm{cm}, \beta=7.22 \times 10^{3} \mathrm{dyne} / \mathrm{cm}$ [14].

\section{B. Piezoelectricity}

The strain in quantum dot causes the atoms in the structure to change the crystal lattice geometry. These changes lead to the polarization. The piezoelectricity is defined as the generation of electric polarization by the application of stress to a crystal lacking a center of symmetry [15], [16]. The piezoelectric potential is obtained by solving Poisson's equation given by

$$
\nabla^{2} V_{P}=\frac{\rho}{\varepsilon(r)}
$$

where $\varepsilon(r)$ is the static dielectric constant of the respective material at the position $r . \rho$ is the piezoelectric charge. The resulting piezoelectric potential $V_{P}$ is included in the tight-binding model as the on site-diagonal potential energy $V_{\text {piezo }}=-e V_{P}$, e is the electron charge. $s p^{3} s^{*}$

\section{Empirical Tight-Binding Method}

We employ $s p^{3} s^{*}$ empirical tight-binding method to calculate the electronic structure of quantum dot. The empirical tight-binding method is represented in terms of an orthogonal basis set of atomic-like orbitals. In our model, the interaction is restricted to the on-site and the nearest neighbours. The tight-binding wave function $\Psi(r)$ is expressed as a linear combination of the atomic orbitals $\phi\left(r-R_{J}\right)$ as given by.

$$
\Psi(r)=\sum_{J} \sum_{\alpha} c_{\alpha, J} \phi_{\alpha}\left(e-R_{J}\right)
$$

Here $R_{J}$ denotes the unit cell and $\alpha$ is described by orbitals $\left(s, p_{x}, p_{y}, p_{z}, s^{*}\right)$. Then the Hamiltonian matrix elements are given by

$$
t_{\alpha^{\prime} R_{J}^{\prime}, \alpha R_{J}}=\left\langle\phi_{\alpha^{\prime}}\left(r-R_{J}^{\prime}\right)|H| \phi_{\alpha}\left(r-R_{J}\right)\right\rangle
$$

These matrix elements $t_{\alpha R_{J}^{\prime}, \alpha R_{J}}$ are treated as the empirical parameters. These parameters are obtained by fitting the bulk-band structure to experimentally known band gaps and effective masses. The fitting parameters are taken from P. Vogl [17].

The parameter for the valence-band offset $\left(E_{v}\right)$ between quantum dot and matrix has to be included in the tight-binding model. This means that for GaSb surrounded by a GaAs matrix, all diagonal matrix elements should be shifted by $\mathrm{E}_{\mathrm{v}}$ compared to the bulk GaSb diagonal matrix elements. In this model, we have performed calculations with the valence-band offsets of $E_{v}=+0.44 \mathrm{eV}$ [2].

Furthermore, in a heterostructure of two materials with different lattice constants, strain effects have to be included for a realistic description of the electronic states. The changes due to the strain are treated on only scaling of the inter-site matrix elements. In general, a relation is given by

$$
t_{\alpha^{\prime} R_{J}^{\prime}, \alpha R_{J}}=\left\langle\phi_{\alpha^{\prime}}\left(r-R_{J}^{\prime}\right)|H| \phi_{\alpha}\left(r-R_{J}\right)\right\rangle\left(\frac{d_{R_{J}^{\prime} R_{J}}^{0}}{d_{R_{J}^{\prime} R_{J}}}\right)^{n_{k 1}}
$$

where $d_{R_{J}^{\prime} R_{J}}^{0}$ and $d_{R_{J}^{\prime} R_{J}}$ are the bond length between the atomic positions of the unstrained and strained materials, respectively. From Harrison's d ${ }^{-2}$ rule [18], [19], $n_{k 1}=2.0$. The validity has been also demonstrated for II-IV materials by S. Sapra [20].

\section{RESUlts AND Discussion}

\section{A. Strain Distribution and Piezoelectricity}

The strain distribution and the piezoelectricity in pyramidal $\mathrm{GaSb} / \mathrm{GaAs}$ quantum dot are analyzed. We consider a GaSb base width of $10.0 \mathrm{~nm}$ and height of $3.6 \mathrm{~nm}$ which is corresponding to the experimental structure [21]. Due to the lattice mismatch between quantum dot and substrate, there is the strain energy inside this system. To minimize the strain energy, we employ the atomistic elasticity method, Valence Force Field Approach. The strain energy is minimized by the conjugate gradient method to obtain the relaxed atomic positions of the quantum dot.

The 2D plots of the strain tensors $e_{x x}, e_{y y}$ and $e_{z z}$ are visualized in Fig. 1 - Fig. 3, respectively. $e_{x x}$ and $e_{y y}$ are negative in the dot region and become positive at the base and the top of quantum dot because GaAs substrate compresses the GaSb dot. $e_{z z}$ is positive at the base of quantum dot. With increasing height within the dot, $\mathrm{e}_{\mathrm{zz}}$ changes its sign and becomes negative at the top of the dot. 


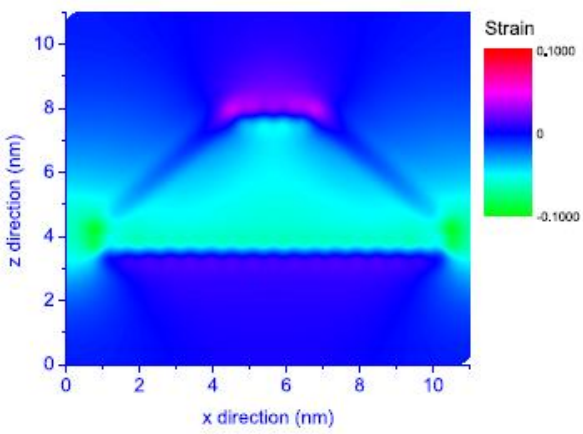

Fig. 1.2D plot of $e_{x x}$.

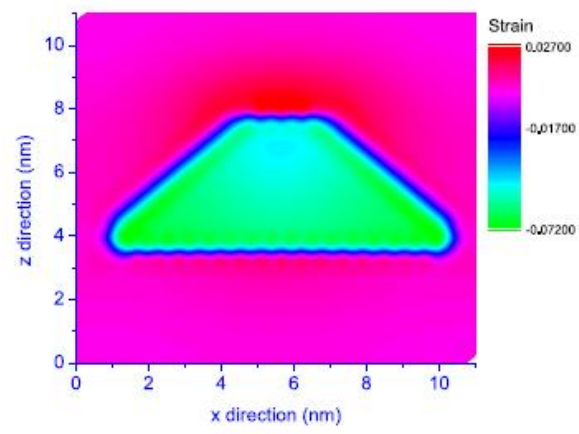

Fig. 2. 2D plot of $e_{y y}$.

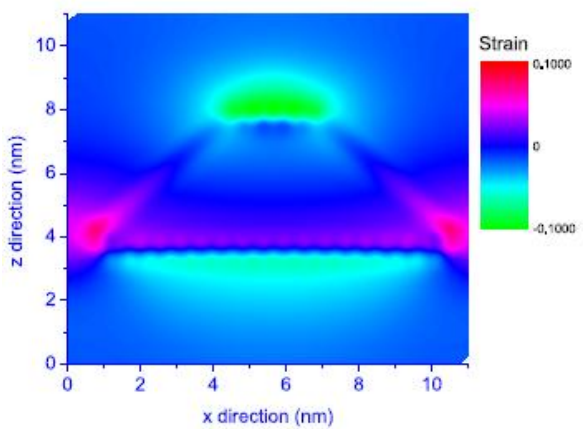

Fig. 3. 2D plot of $e_{z z}$

Apart from the strain distribution, we have also calculated the piezoelectric potential in $\mathrm{GaSb} / \mathrm{GaAs}$ quantum dot by solving the corresponding 3D Poisson equation. The iso-surface of the piezoelectric potential is plotted in Fig. 4. It has shown that the piezoelectric potential is concentrated in the region close to the top of the pyramid. Below the base of the quantum dot, the potential is relatively weak, which is not shown owing to its smaller values. It expresses that the piezoelectric potential is mostly positive (red iso-surface) along the [110] direction and negative (blue iso-surface) along the [110] direction.

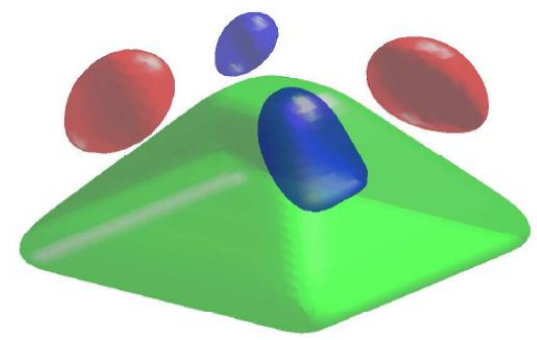

Fig. 4. 3D plot of the piezoelectric potential.

\section{B. Electronic Structure}

The energy states are computed as a function of the dot size using the empirical $s p^{3} s^{*}$ tight-binding method. The shape of quantum dot is pyramidal. The base width is $\mathrm{b} \mathrm{nm}$ and the height of the dot is $b / 2 \mathrm{~nm}$. We have calculated the first-two states of the electron and hole states as a function of the dot sizes in Fig. 5. The graph shows that the electron states remain almost constant with increasing the dot size. In contrast, the hole states become increasing when the dot sizes become large. Therefore, the band gap of $\mathrm{GaSb} / \mathrm{GaAs}$ quantum dot becomes smaller as a function of the increasing dot size. The calculations are consistent with the quantum confinement.

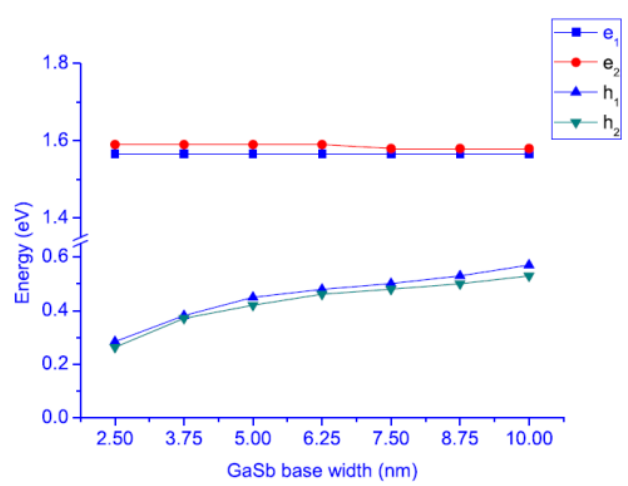

Fig. 5. The energy spectrum $v s$ GaSb dot sizes in GaAs matrix.

Next we plot the 3D wave functions of the electron and hole states. We consider a GaSb base width of $10.0 \mathrm{~nm}$ and height of $3.6 \mathrm{~nm}$ embedded in GaAs matrix. The iso-surfaces of the wave functions demonstrate that the electrons are not localized in $\mathrm{GaSb}$ dot while the holes are mainly confined in the dot region as shown in Fig. 6 and 7, correspondingly. The green and red isosurface levels are selected as quantum dot shape and 0.75 of the maximum probability density, respectively. These calculations confirm that the band alignment of $\mathrm{GaSb} / \mathrm{GaAs}$ quantum dot is type-II.

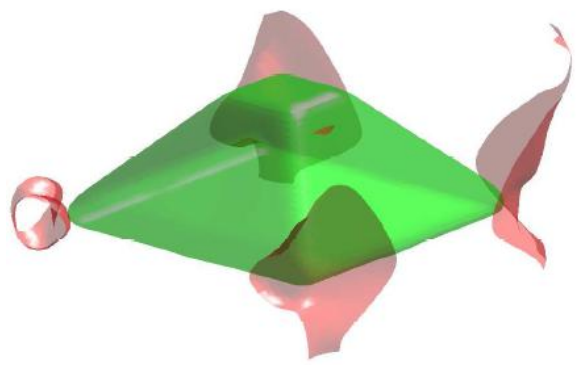

Fig. 6. 1st electron wave function.

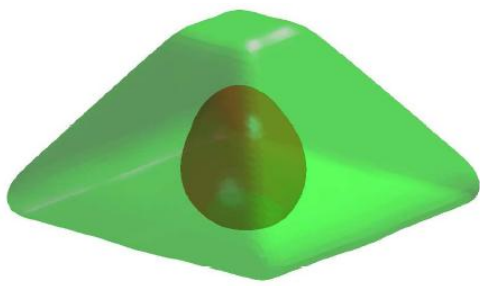

Fig. 7. 1st hole wave function.

After studying the theoretical calculations of $\mathrm{GaSb} / \mathrm{GaAs}$ quantum dot, we then compare our model with the experiment and theoretical method. For this purpose, we compare our results with reported data. Muller-Kirsch and co-authors [21] report PL spectra from several $\mathrm{GaSb} / \mathrm{GaAs}$ 
quantum dot sizes. In this comparison, we compare the PL spectra of the pure $\mathrm{GaSb}$ dot which has the average base width of $10.00 \mathrm{~nm}$. The result demonstrates that theoretical PL peak is at $1.24 \mathrm{eV}$ and experimental PL peak is approximately $1.15 \mathrm{eV}$. Our PL calculation is quite consistent with experiment. Kamil Gradkowski and co-authors [2] have compared the ground-state transition energy from the photoluminescence (PL) measurements of the $\mathrm{GaSb} / \mathrm{GaAs}$ quantum dot structure without an $\operatorname{In}_{0.14} \mathrm{Ga}_{0.86}$ As capping quantum well with the 8-band k.p method. The base dimension is $10 \mathrm{~nm} \times 10 \mathrm{~nm}$ and height is $3 \mathrm{~nm}$. The ground-state transition energy from 8-band k.p approach is $1.16 \mathrm{eV}$ and $1.04 \mathrm{eV}$ is obtained from the $\mathrm{sp}^{3} \mathrm{~s}^{*}$ empirical tight-binding model. Therefore, the tight-binding calculation is quite consistent with k.p method.

\section{CONCLUSION}

We have calculated the strain distribution in a typical self-assembled $\mathrm{GaSb} / \mathrm{GaAs}$ dot by the means of the Valence Force Field Approach. We have also achieved to employ the $s p^{3} s^{*}$ empirical tight-binding theory to numerically study electronic properties of $\mathrm{GaSb} / \mathrm{GaAs}$ self-assembled quantum dots. GaSb/GaAs quantum dot exhibits a type-II band alignment. The results demonstrate that the band gap become smaller as the increasing dot size. Our investigation shows that confinement exists for only the hole states. Finally, the PL calculation is quite consistent with experiment and k.p approach.

\section{ACKNOWLEDGMENT}

I would like to acknowledge Department of Physics Faculty of Science, Ubon Ratchathani University, Ubon Ratchathani.

\section{REFERENCES}

[1] Tatebayashi, J. Balakrishnan, G. Huang, S. H. Khoshakhlagh, A. Mehta, M. Dawson, and L. R. Huffaker, "Optical Properties of Stranski-Krastanow and Strain-Free GaSb Quantum Dots on GaAs Substrates - Towards Sb based Type-II Quantum Dot Emitters Nanotechnology," in Proc. Sixth IEEE Conference on NANO, vol. 119, 2006.

[2] K. Gradkowski et al., Journal of Luminescence, vol. 129, pp. 456, 2009.

[3] M. Hayne et al., "Optically induced charging effects in self-assembled GaSb/GaAs quantum dots," Phys. Rev. B, vol. 70, pp. 081302, 2004.

[4] A. Schliwa, O. Stier, D. Bimberg, H. Kirmse, L. Mller-Kirsch, R. Heitz, and W. Neumann, "Many-particle effects in type-II quantum dots," Appl. Phys. Lett, vol. 78, pp. 1418, 2001.
[5] U. W. Pohl, D. Bimberg, I. Hsler, H. Kirmse, L. Mller-Kirsch, R. Heitz, and W. Neumann, "Temporal evolution of GaSb/GaAs quantum dot formation," Appl. Phys. Lett, vol. 79, pp. 1027, 2001.

[6] C. Pryor, J. Kim, L. W. Wang, A. J. William, and A. Zunger, "Comparison of two methods for describing the strain profiles in quantum dots," J Appl. Phys., vol. 83, pp. 2548, 1998.

[7] H.-T. Jiang and J. Singh, "Strain distribution and electronic spectra of InAs/GaAs self-assembled dots: An eight-band study," Phys. Rev. B, 56, pp. 4696, 1997.

[8] P. N. Keating, "Effect of Invariance Requirements on the Elastic Strain Energy of Crystals with Application to the Diamond Structure," Phys. Rev., vol. 145, pp. 637-645, 1966.

[9] M. Grundmann, O. Stier, and D. Bimberg, "Electronic and optical properties of strained quantum dots modeled by 8-band kp theory," Phys. Rev. B, vol. 59, pp. 688-5701, 1999.

[10] J. Kim, L.-W. Wang, and A. Zunger, "Electronic structures of [110]-faceted self-assembled pyramidal InAs/GaAs quantum dots," Phys. Rev. B, vol. 59, pp. 5678-5687, 1999.

[11] Y. M. Niquet, G. Allan, and C. Delerue, "Quantum confinement energies in zinc-blende IIIV and group IV semiconductors," Appl. Phys. Lett, vol. 77, pp. 639, 2000.

[12] S. Schulz and G. Czycholl, "Tight-binding model for semiconductor nanostructures," Phys. Rev. B, vol. 72, pp. 165317, 2005.

[13] S. M. North, P. R. Briddon, M. A. Cusack, and M. Jaros, "Electronic structure of GaSb/GaAs quantum domes," Phys. Rev. B, vol. 58, pp. 12601 - 12604, 1998.

[14] R. M. Martin, "Elastic Properties of ZnS Structure Semiconductors," Phys. Rev. B, vol. 1, pp. 4005-4011, 1970.

[15] J. H. Davies, "Elastic and piezoelectric fields around a buried quantumbdot: A simple picture," J. Appl. Phys., vol. 84, pp. 1358, 1998.

[16] G. Bester and A. Zunger, "Cylindrically shaped zinc-blende semiconductor quantum dots do not have cylindrical symmetry: Atomistic symmetry, atomic relaxation, and piezoelectric effects,' Phys. Rev. B, vol. 71, pp. 045318, 2005.

[17] P. Vogl, H. P. Hjalmarson, and J. D. Dow, "A semi-empirical tight-binding theory of the electronic structure of semiconductor," $J$. Phys. Chem. Solids, vol. 44, pp. 365, 1983.

[18] S. Schulz and G. Czycholl, "Tight-binding model for semiconductor nanostructures," Phys. Rev. B, vol. 72, pp. 165317, 2005.

[19] W. A. Harrison, Elementary Electronic Structure, World Scientific Publishing Company, 1999.

[20] S. Sapra, N. Shanthi, and D. D. Sarma, "Realistic tight-binding model for the electronic structure of II-VI semiconductors," Phys. Rev. B, vol. 66, pp. 205202, 2002.

[21] L. Muller-Kirsch et al., "Temporal evolution of GaSb/GaAs quantum dot formation," Appl. Phys. Lett. vol. 79, pp. 1027, 2001.

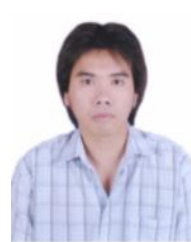

Worasak Sukkabot received a B.S. in Physics from the Ubon Ratchathani University in 2003 and a Ph.D. in Physics from University of Surrey in 2010. He did postdoctoral research at National Chiao Tung University, Taiwan, in 2011 before joining the faculty in the Department of Physics at the Ubon Ratchathani University, Thailand, in 2011. He is currently a lecturer in Department of Physics at the Ubon Ratchathani University. His main research interest is in the theoretical area of low-dimensional semiconductor nanostructures. He focuses on the electronic structure and optical properties of self-assembled quantum dots and nanocrystal by implementing the tight-binding methods. 